\title{
Formation interdisciplinaire à la pédagogie dans l'enseignement supérieur : de l'expérience individuelle à la dynamique collective pour impulser une transformation pédagogique
}

Catherine de la Fouchardière, Laure Abiad, Moussa Laanani, Marine Lanteri, Morgane Maridet, Annabelle Tenenbaum and Isabelle Colombet

\section{(2) OpenEdition}

\section{Journals}

Electronic version

URL: https://journals.openedition.org/ripes/3609

DOI: $10.4000 /$ ripes.3609

ISSN: 2076-8427

Publisher

Association internationale de pédagogie universitaire

Electronic reference

Catherine de la Fouchardière, Laure Abiad, Moussa Laanani, Marine Lanteri, Morgane Maridet, Annabelle Tenenbaum and Isabelle Colombet, "Formation interdisciplinaire à la pédagogie dans l'enseignement supérieur : de l'expérience individuelle à la dynamique collective pour impulser une transformation pédagogique", Revue internationale de pédagogie de l'enseignement supérieur [Online], 37(3) | 2021, Online since 16 November 2021, connection on 27 September 2022. URL: http:// journals.openedition.org/ripes/3609; DOI: https://doi.org/10.4000/ripes.3609

This text was automatically generated on 27 September 2022

Creative Commons - Attribution-NonCommercial-ShareAlike 4.0 International - CC BY-NC-SA 4.0 https://creativecommons.org/licenses/by-nc-sa/4.0/ 


\title{
Formation interdisciplinaire à la pédagogie dans l'enseignement supérieur : de l'expérience individuelle à la dynamique collective pour impulser une transformation pédagogique
}

\author{
Catherine de la Fouchardière, Laure Abiad, Moussa Laanani, Marine \\ Lanteri, Morgane Maridet, Annabelle Tenenbaum and Isabelle Colombet
}

\section{Remerciements}

Nous remercions l'ensemble des certifiés 2018 : Laure Abiad, Isabelle Colombet, Catherine de la Fouchardière, Moussa Laanani, Natacha Le Fur, Odile Racine, Esther Rogan et Annabelle Tenenbaum.

Nous tenons à remercier également l'ensemble de l'équipe SAPIENS et particulièrement, Marine Lanteri et Morgane Maridet pour avoir dirigé l'entretien de groupe et pour leurs contributions à l'écriture de cet article.

\section{Introduction et contexte}

En France, on observe une grande hétérogénéité des pratiques enseignantes, structurée essentiellement par les différentes exigences disciplinaires. Les enseignants développent ainsi différentes postures, comme définies par Deschryver et Lameul (2016) en fonction de leur discipline et du rapport aux savoirs qu'elle implique. L'enseignement consiste essentiellement à faire entrer les étudiants dans certains types de raisonnements, activités, relativement standardisés et faisant référence pour la discipline, sans réellement partir des acquis et des besoins d'apprentissage des étudiants, qui ont pourtant évolué en même temps que la société dans laquelle ils 
s'insèrent. Jusque récemment, aucune formation à la pédagogie n'était exigible pour la qualification des enseignants universitaires et les activités de recherche restaient plus valorisées que les activités d'enseignement dans la carrière des enseignantschercheurs.

Cependant, depuis le début des années 2010, les références à la pédagogie sont nombreuses dans le discours des instances gouvernementales invoquant la nécessité de formation aux pratiques pédagogiques (Lalle et Bonnafous, 2019). Le décret du 9 mai 2017 instaure l'obligation de formation à la pédagogie pour les nouveaux enseignantschercheurs (Journal officiel de la République française, 2017). En 2018, François Taddéi souligne l'importance de la collaboration dans l'apprentissage et l'urgence pour la recherche et l'éducation d'évoluer en synergie dans un monde en pleine mutation. De nouveaux formats apparaissent, comme, par exemple, celui du MOOC, qui permettent aux enseignants de se former à leur rythme (MOOC « Se former pour enseigner dans le supérieur », s.d.).

On observe donc une mobilisation relative des différents acteurs portant sur la réflexion puis la mise en œuvre de la transformation pédagogique. La volonté du Ministère est de favoriser les initiatives, de les mettre en avant afin qu'elles puissent inspirer (Pratiques pédagogiques avec le numérique, s.d.). Pourtant, il semble à ce jour que les enseignants formés à la pédagogie sont encore peu nombreux et que les vocations sont individuelles plus que dictées par une politique institutionnelle.

La Communauté d'Universités Sorbonne Paris Cité (USPC) s'est dotée en 2014 du Service d'Accompagnement aux Pédagogies Innovantes et à l'Enseignement Numérique de Sorbonne Paris-Cité (SAPIENS), adossé au CRI (Centre de Recherches Interdisciplinaires) et partageant ses locaux, ayant pour mission d'agir à la transformation des pratiques pédagogiques en interdisciplinarité. À partir de septembre 2016, cette équipe a conçu une offre de formation modulaire, à destination commune des doctorants, des enseignants chercheurs (EC) et des chargés de cours. En juin 2017, huit EC et chargés de cours ayant suivi la totalité des ateliers proposés, se sont vu proposer de compléter leur formation par un accompagnement plus individualisé afin d'opérer une transformation pédagogique d'un de leur cours (préparation, observation d'une séance et débriefing). En août 2018, chaque enseignant a rédigé un bilan réflexif de l'ensemble de son expérience de formation et de transformation pédagogique. L'expérience de transformation pédagogique et la rédaction du bilan réflexif ont concouru à l'obtention du nouvellement créé CertifiENS, formule innovante en France de certificat de pédagogie.

Nous constituons un sous-groupe de cette première promotion d'enseignants certifiés, poursuivant une démarche de communauté de pratiques (Wenger, 1998). Nous avons souhaité rendre compte de cette expérience de formation continue, avec l'aide de deux des formatrices de l'équipe SAPIENS qui nous ont accompagnés dans notre parcours pour favoriser l'explicitation de notre analyse réflexive. Cette expérience nous a convaincus qu'une transformation des pratiques pédagogiques réfléchie et conduite en interdisciplinarité pouvait être un levier pour envisager une transformation plus globale et effectivement opérante sur le terrain.

L'objectif de cet article est de décrire notre expérience de formation et de transformation pédagogique puis d'en proposer des éléments d'analyse réflexive individuelle puis collective, afin de montrer comment l'engagement dans cette 
formation, parti de motivations propres à chaque enseignant, a fait émerger une dynamique de construction collective.

Nous proposons donc, dans une première partie descriptive, de présenter 1) les caractéristiques de notre groupe pour en souligner l'interdisciplinarité, 2) l'expérience de formation modulaire et de transformation pédagogique et 3) les méthodes utilisées pour expliciter l'analyse réflexive de cette expérience, au niveau individuel (bilans réflexifs rédigés par chacun de nous pour valider sa formation) et collectif (entretien de groupe). Dans une deuxième partie plus réflexive, nous présenterons les résultats de ces analyses, avec les principaux éléments des bilans réflexifs et les résultats issus de nos travaux de groupe. Enfin dans une troisième partie, nous envisagerons les suites données à cette expérience, dans le contexte individuel et institutionnel de chacun et les perspectives de poursuite d'une communauté de pratiques.

\section{Descriptif de l'expérience de formation CertifiENS}

\subsection{Description de la première promotion CertifiENS}

La particularité de la première promotion Certifiens réside dans une grande hétérogénéité, que ce soit en termes de rattachement universitaire, de statut ou de discipline enseignée. Nous sommes issus de six établissements d'enseignement supérieur distincts dont quatre universités et deux instituts, tous situés à Paris; nous enseignons dans cinq filières majeures: gestion-marketing, langues, sciences de la santé, sciences politiques et statistique-informatique décisionnelle. Le temps imparti à l'enseignement diffère pour chacun, en fonction de sa filière et de ses autres fonctions : de recherche, hospitalière. Le poids de l'enseignement n'est ainsi pas réparti de la même manière en fonction du nombre de missions auxquelles certains d'entre nous doivent répondre. Pour ceux en filière médicale (statut hospitalo-universitaire), ayant à charge ces trois missions, la mission d'enseignement est bien souvent la moins reconnue et le temps qui lui est alloué est moindre.

Nous sommes également différents autant par nos statuts et ancienneté dans l'exercice de nos fonctions, que par le public à qui nous nous adressons, en termes de niveau d'étude ou de type de formation.

Notre rencontre a révélé à chacun la force de l'interdisciplinarité, les uns découvrant dans l'univers académique et pédagogique des autres de quoi faire évoluer réflexions et mises en pratique. Les prérequis universitaires et compétences nécessaires à un poste d'enseignant sont variables d'une filière à une autre et malgré des expériences et anciennetés d'enseignement variés, aucun de nous n'avait bénéficié d'une formation à la pédagogie active, le constat commun effectué étant la mise en œuvre par tous d'un enseignement selon un modèle classique de pédagogie transmissive de connaissances.

\subsection{Expérience de la formation interdisciplinaire}

La formation modulaire que nous avons suivie présentée en annexe 1, a permis une première approche des principaux concepts pédagogiques. Condensée sur un an, à raison de quatre à six ateliers par trimestre à choisir selon ses besoins et disponibilités, chacun déclinant un concept sur un mode de pédagogie active, cette offre s'est avérée particulièrement invitante et adaptée, pour les enseignants déjà en activité que nous 
étions, introduisant même par l'exemple une méthode pédagogique nouvelle par rapport à nos pratiques courantes.

A la fin, ou en parallèle de cette formation, les conseillères pédagogiques de l'équipe SAPIENS, ont proposé à chaque candidat à la certification, de les accompagner pour conduire une transformation pédagogique d'un de leur cours. Cet accompagnement a consisté a minima en une réunion de préparation avec production de documents pédagogiques (par exemple un scénario de cours), l'observation par la conseillère de la séance de cours transformé et une réunion de relecture critique de la séance de cours et d'évaluation visant à ajuster le résultat de cette transformation.

Les principaux ateliers des quatre modules de formation, jugés nécessaires pour maîtriser les concepts-clé étaient: alignement pédagogique, motiver les étudiants, définir les objectifs du cours, rédiger un syllabus, scénariser son cours, évaluer ses étudiants). Nous avons pu ensuite mobiliser ces concepts au cours de notre travail de transformation pédagogique accompagné par l'équipe SAPIENS.

Ainsi, ce programme de formation a pu s'enrichir des échanges et des discussions en interdisciplinarité lors de séances de formations (travail sur des syllabus ou des grilles critériées, par exemple), dans la mesure où le calendrier de suivi des ateliers de formation était laissé au libre choix des candidats (les moins disponibles pouvant les suivre sur deux ans) et où les transformations pédagogiques accompagnées pouvaient être conduites en parallèle des ateliers de formation.

\subsection{Méthode d'analyse réflexive de l'expérience}

\subsubsection{Au niveau individuel}

Dans le cadre de l'obtention du certificat CertifiENS, nous avons rédigé un bilan réflexif décrivant la transformation pédagogique opérée au sein d'un de nos enseignements, de sa conception à sa mise en œuvre, pour finalement poursuivre ce processus de transformation. Ce bilan était évalué selon une grille critériée, fournie par l'équipe SAPIENS en amont de la rédaction, qui comportait deux axes: une dimension qui évaluait ce qui avait été mis en place lors du cours choisi pour l'accompagnement et une autre dimension qui évaluait spécifiquement le bilan rédigé sur la transformation pédagogique opérée. Avec ses quatre niveaux d'évaluation: "à développer - en développement - au seuil d'entrée - au-delà des attentes", cette grille évaluait le réinvestissement des concepts pédagogiques phares: les objectifs - le syllabus l'alignement pédagogique - la scénarisation - les activités centrées sur les apprentissages et l'évaluation. Concernant les critères spécifiques à la forme du bilan, il s'agissait d'évaluer le respect du format (avec six rubriques : objectifs pédagogiques contexte du cours transformé - construction et transformation du cours - mise en pratique et analyse - pistes de modifications - pistes d'évolution et annexes) - les ressources théoriques mobilisées et l'explicitation du processus de construction ou de transformation de cours. Cette grille détaillée nous a guidés pas à pas dans l'élaboration de notre pratique réflexive et a ainsi facilité le travail de rédaction du bilan. Elle nous a permis également de lire de façon éclairée les bilans des autres certifiés.

Partant de la relecture de chacun de nos bilans réflexifs, nous proposons en deuxième partie d'en dégager le processus d'appropriation et de mobilisation des principaux concepts pédagogiques expérimentés dans nos transformations pédagogiques 
respectives, afin, en synthèse et perspectives, de souligner les éléments qui ont ensuite favorisé notre engagement dans une dynamique plus collective.

\subsubsection{Au niveau collectif}

Pour rendre compte d'une analyse réflexive collective de notre expérience de formation, nous avons sollicité les conseillères pédagogiques de SAPIENS. Également chercheures en sciences sociales, formées aux techniques de l'entretien d'explicitation (Vermersch, 2017), elles ont conçu la méthodologie de l'analyse réflexive et mis en place un entretien collectif ou focus group ${ }^{1}$ qui a permis de faire émerger nos motivations et intentions individuelles initiales, mais également la dynamique collective de transformation des pratiques pédagogiques.

Pour répondre à notre sollicitation, elles ont choisi de s'aligner avec l'approche pédagogique proposée dans les ateliers de formation SAPIENS et de se rapprocher d'une démarche de recherche ancrée dans un paradigme socio-constructiviste. En effet, si l'entretien individuel a pour objet principal « le discours du sujet » et est un «acte de communication " oral (De Ketele et Roegiers, 2015), la particularité des entretiens collectifs est que le discours émerge, non pas d'un seul acteur, mais des interactions entre plusieurs individus. Le chercheur s'engage avec les participants dans le processus de construction de la recherche à travers la discussion qui se créée autour des réponses aux questions (Casey, 1995; Herda, 1999). Ancré dans ce paradigme de recherche, le chercheur voit une remise en question de sa posture: il ne dispose plus d'un regard extérieur privilégié, mais doit guider la conversation afin qu'elle soit riche et productive (Moyle, 2007). Contrairement à son utilisation courante en psychosociologie ou en intervention sociologique (Touraine, 1978), où l'un de ses objectifs est la transformation des représentations et des pratiques des individus impliqués dans le groupe, les chercheures ont souhaité avant tout s'appuyer sur les interactions pour recueillir des informations et amener les participantes à développer leurs points de vue. Par ailleurs, le focus group semblait être un format intéressant pour faciliter l'autoanalyse des participantes amenées à confronter leur expérience à celles de leurs collègues.

L'intention de cet exercice était de proposer un moment d'échange pour nous permettre de verbaliser et ainsi de conscientiser notre expérience d'apprentissage dans le cadre de CertifiENS. L'objectif de cet entretien collectif était multiple : d'une part il s'agissait de pouvoir recueillir des données qualitatives concernant notre expérience de participation à CertifiENS, d'identifier quelles avaient pu être nos intentions et motivations initiales, mais également de se poser la question de l'interdisciplinarité (ses avantages et ses limites dans le cadre du développement professionnel des enseignants-chercheurs) et finalement, d'identifier de potentielles pistes d'amélioration pour le parcours de formation CertifieNs afin de toujours mieux accompagner les enseignants dans leur processus d'évolution pédagogique.

Cet entretien collectif de type semi-directif a été mené au mois de mars 2019, six mois après la validation de CertifiENS par la promotion 2018. Il a été conduit avec un groupe représentatif de l'interdisciplinarité et de l'hétérogénéité des enseignants certifiés : quatre des huit enseignants ayant validé le certificat cette année-là étaient présents. Un guide d'entretien (Annexe 2) a été élaboré et rédigé en amont par les deux chercheures qui ont animé puis analysé cet entretien, qu'elles ont également enregistré et retranscrit elles-mêmes. 


\section{Bilan et analyse d'une expérience collective de transformation pédagogique en interdisciplinarité}

\subsection{Analyse individuelle tirée des bilans réflexifs}

\subsubsection{Une remise en question profonde de ses pratiques}

La pratique réflexive nécessaire à toute transformation pédagogique fait l'objet de deux formations dans le cursus que nous avons suivi: une formation "questionner mes pratiques » en début de cycle et une en fin de cycle. Ce travail préparatoire permet d'analyser sa pratique pédagogique actuelle et il est intéressant de constater que les questions soulevées n'ont pas la même résonance pour tous. Les encadrés suivants témoignent à ce titre de la variété de nos attentes et besoins et laissent entrevoir la richesse qu'a pu constituer notre interdisciplinarité.

CF : Transformer son enseignement prend beaucoup de temps. Cela oblige à une auto-analyse et une remise en question de ses pratiques.

- Comment je me vois en tant qu'enseignant? Quel est mon rôle?

- Mes étudiants sont-ils intéressés et motivés pendant mon cours?

- Comment puis-je améliorer leur interaction et leur participation?

- Sur quels points (méthodologiques, pédagogiques, format du cours...) dois-je agir de façon prioritaire?

LA : Enseignant le français langue étrangère, les questionnements sur ma capacité à interagir et à créer une dynamique motivationnelle en cours m'interpelaient moins que les points méthodologiques que je voulais retravailler. Comment aligner son évaluation sur les contenus? Comment favoriser un apprentissage plus en profondeur? Comment construire une grille critériée. J'avais besoin d'être guidée d'un point de vue méthodologique, plus que sur mes compétences à animer ou à favoriser l'interaction dans mes cours.

Le questionnement de nos pratiques pédagogiques, à la fois individuellement, puis en collectif, lors de nos ateliers, a été le point de départ de nos transformations respectives. Il a révélé une volonté commune, d'une part d'entrer dans un processus de réflexivité afin de développer nos compétences professionnelles, d'autre part de se concentrer sur ce qui nous rassemble en pédagogie en prenant en compte notre diversité disciplinaire.

\subsubsection{Processus de transformation opérée par la mobilisation des concepts pédagogiques enseignés}

En procédant à une lecture croisée de nos bilans respectifs, il est apparu clairement que les concepts clés en pédagogie avaient été réinvestis par tous. Nous avons tous réfléchi aux objectifs de nos cours, puis, plus précisément, en travaillant la scénarisation de nos séances, aux objectifs de chacune des séances. La notion d'alignement pédagogique nous est apparue comme une notion nouvelle et absolument centrale dans la transformation de nos pratiques. 


\section{Alignement pédagogique}

L'alignement pédagogique (Biggs, 1996) est le concept principal dont nous nous sommes saisis, avec un effet disruptif pour la plupart de nos enseignements. Étant tous installés dans une conception de nos cours de manière transmissive d'un contenu logique, à l'issue de la séance de formation sur l'alignement pédagogique, l'approche s'est inversée. En effet, il s'agissait de partir, non pas de ce que nous avions à transmettre, mais d'un questionnement sur ce que les étudiants devaient avoir appris ou acquis en termes de savoirs ou savoir-faire, puis de construire des activités d'apprentissage et des évaluations permettant de suivre leur progression. Comme l'intégrait ML dans son bilan, «le respect de l'alignement pédagogique doit guider l'ensemble de la conception et de la conduite du cours, après avoir établi des objectifs pédagogiques solidement définis ". À l'échelle d'un cours sur un semestre, la mise en application de ce concept ne s'est néanmoins pas toujours faite sans difficulté, comme l'illustre l'exemple suivant, d'un cours magistral sur les métiers de l'enseignement destiné aux étudiants de L1 à L3.

LA : Le cours intitulé «métiers de l'enseignement » avait pour objectif principal de présenter un panorama des différents métiers de l'enseignement vers lesquels pourraient s'orienter les étudiants. Pour atteindre cet objectif, je souhaitais qu'ils soient capables, à la fin du semestre d'identifier les différents métiers de l'enseignement et les formations correspondantes, de questionner des professionnels de l'enseignement à travers un entretien, ce qui leur permettrait de réfléchir ensuite à leur orientation professionnelle.

Forte de mes nouvelles connaissances sur l'alignement pédagogique, je n'ai pas eu de difficultés à construire des activités pédagogiques diversifiées et interactives correspondant aux deux premiers objectifs : « identifier les différents métiers » et « s'entretenir avec des professionnels de l'enseignement». En revanche, il a été beaucoup plus complexe d'élaborer des stratégies d'évaluation pertinentes pour le troisième objectif " réfléchir à son orientation professionnelle ", qui est difficilement mesurable: comment évaluer la réflexion de l'étudiant concernant son orientation professionnelle si ce n'est, poser la question suivante lors d'une Évaluation des Enseignements par les Étudiants : "En quoi cette UE vous a permis de préciser votre orientation professionnelle?". J'ai éprouvé une sorte de frustration de ne pas pouvoir construire un alignement pédagogique cohérent pour ce dernier objectif.

\section{objectifs de cours}

Alors que la définition des objectifs pédagogiques (Mager, 1972; Nadeau, 1988; De Ketele et al., 2007) est un concept clé, nous nous sommes rapidement aperçus, lors de ce module de formation, que la formulation d'objectifs clairs et explicites n'était pas un exercice aisé, les relier avec les évaluations l'était encore moins. Cette formation a été l'occasion de rappeler également que définir ses objectifs, c'est créer un socle pour choisir ensuite des activités d'apprentissages adaptées.

Ainsi, lors de nos échanges en petits groupes lors de la formation "définir les objectifs ", l'exercice de formulation des objectifs amenait à expliquer son cours à des collègues "naïfs ", découvrant une autre discipline et donc particulièrement attentifs et contributifs sur la forme, levier pour comprendre le contenu. Plusieurs repères ont ainsi émergé comme particulièrement structurants de la transformation future de nos pratiques: le questionnement des objectifs de cours déjà anciens et installés dans une certaine routine, avec la grille SMART (Spécifique, Mesurable, Atteignable, Réaliste, 
Temporel), la reconnaissance et l'acceptation d'un nécessaire deuil de certains contenus et enfin l'explicitation des objectifs dès le début du semestre et à chaque début de séance pour favoriser l'interactivité et l'implication active des apprenants dans des activités pédagogiques alignées aux objectifs et en améliorant ainsi l'efficacité. IC : La réflexion sur les objectifs dans le cadre d'une UE de master 2 recherche en santé publique (3 séances de 3h) a conduit à redéfinir une scénarisation du cours plus adaptée à un groupe de 15-20 étudiants et a permis de formuler des objectifs permettant d'accéder aux niveaux supérieurs de la taxonomie de Bloom: alors qu'auparavant, les activités essentiellement transmissives de contenu et échange en grand groupe sur des lectures d'articles ne permettaient d'atteindre que des objectifs de l'ordre des "connaissances" ou de "compréhension", des activités plus structurées et rythmées par petits groupes autour des étapes d'élaboration d'un projet de recherche ont permis de hisser les efforts des étudiants sur des objectifs d'" application ", d'" analyse ", voire d'" évaluation » et de "synthèse ».

\section{Scénarisation}

La séance intitulée "scénariser mon enseignement» suit celle sur "questionner ses pratiques pédagogiques " et "définir ses objectifs ». Sa position, dès l'introduction, suggère bien qu'elle fait partie intégrante, constituante, d'une nouvelle conception de l'enseignement. Ainsi, une fois les objectifs posés, l'enseignant réfléchit à chacune des étapes à la lumière de la scénarisation (Basque, 2007; Daele et Berthiaume, 2013; Musial et al., 2012). Loin d'être une structure à apporter au cours, une fois le contenu et les différentes séances définis, la scénarisation est une manière d'envisager l'élaboration d'un cours qui s'impose rapidement à la fois comme une évidence et comme une nouvelle pratique à intégrer. À quelques deux années de distance de cette formation, la scénarisation fait désormais partie intégrante de notre transformation pédagogique et nous ne pouvons plus concevoir un cours en le dissociant de sa scénarisation.

Notre démarche repose ainsi sur une simultanéité entre atteinte des objectifs, structuration du temps d'enseignement et perpétuelle recherche de stratégies pédagogiques. Ceci vient compenser ces sensations antérieures de lacunes dans l'enseignement, qui n'était réfléchi qu'en fonction du thème et contenu du cours à transmettre et non en termes d'objectifs et de moyens pour les atteindre. De fait, cela sous-entend d'être en réflexion constante sur ses intentions pédagogiques, les conditions d'efficacité et ainsi d'avoir une posture dynamique dans le temps. Le cours est conçu pour être évolutif et répondre aux contextes du moment et des étudiants, il n'est pas élaboré comme un temps statique. Il s'agit de formaliser le déroulement de la séance par des alternances dans les différents temps de cours, des activités diverses et cohérentes entre elles, en pensant pour chacune le temps nécessaire. Cette structuration autour d'un timing bien cadré permet également d'atteindre les objectifs définis a priori. Scénariser son enseignement, c'est donner une logique et un rythme.

\section{Syllabus}

Dans nos activités d'enseignement précédant les formations SAPIENS, les syllabus (Brauer, 2011) étaient soit inexistants soit imposés par les institutions pour lesquelles nous travaillions. La co-construction de nos syllabus respectifs a commencé dès l'atelier SAPIENS au cours duquel l'interdisciplinarité a été à nouveau un levier, permettant des regards croisés d'autant plus critiques et enrichissants. Une attention particulière a été 
portée à l'élaboration du syllabus des cours que nous avons transformés et la notion de " contrat » inhérente au syllabus s'est avérée être un outil intéressant pour impliquer les étudiants dans la formation.

LA : Le syllabus (Annexe 3), que j'ai élaboré dans le cadre du cours sur les métiers de l'enseignement, avec l'aide de l'équipe SAPIENS a été explicité dès le premier cours, ce qui permettait de faire savoir aux étudiants précisément le contenu et les attentes de ce cours. Je m'y suis référée à chaque début de séance afin que les étudiants en apprécient la progression. J'ai pu remarquer qu'ils étaient très attentifs à ce rituel de démarrage et que cela facilitait l'interaction dans ce cours magistral. La clarté des objectifs présentés dans le syllabus a contribué à un meilleur engagement de la part des étudiants.

La lecture croisée de nos bilans a fait apparaître le syllabus comme objet-témoin de notre transformation pédagogique. Elle a permis de mettre en relief l'aspect fondateur de cet outil, pierre angulaire de la relation étudiant - enseignant. Ce contrat, à travers la définition des objectifs, a témoigné de la prise en compte des besoins de nos étudiants, favorisant ainsi une plus grande implication de leur part. Cet engagement réciproque nous a permis de "garder le cap » de notre transformation pédagogique et de provoquer des échanges avec nos pairs.

Cependant, la notion de syllabus est reprise de manière inégale dans les différents bilans, reflétant l'inutilité de ce concept pour un enseignant qui participe pour un cours ou deux à un module d'enseignement sans avoir de responsabilité de l'ensemble ni donc le pouvoir d'action sur une transformation globale, situation fréquente en faculté de médecine par exemple.

\section{Motivation des apprenants}

Pendant la formation SAPIENS sur ce thème, nous avons retenu que, pour encourager la dynamique motivationnelle, l'enseignant n'a de prise que sur les facteurs relatifs aux cours, alors qu'il ne peut pas agir sur les facteurs extérieurs (facteurs relatifs à l'étudiant, à l'établissement, à la société) (Viau, 1999). Cette prise de conscience a permis, dans un premier temps, de diminuer la «pression» qui repose sur nous, enseignants et nous avons pu ainsi nous concentrer, ensuite, uniquement sur les facteurs sur lesquels nous pouvions agir et et cibler notre action. Nous avons réfléchi ensemble à la conception d'activités qui puissent amener chez l'étudiant le sentiment de valeur, de compétence et de contrôlabilité.

LA : Lorsqu'aujourd'hui, j'élabore une scénarisation, je m'appuie sur une grille critériée afin de vérifier que les activités soient suffisamment motivantes, par exemple: dès le début de l'activité, les objectifs d'apprentissage ont-ils bien été présentés et compris par tous les étudiants? L'activité correspond-elle à leurs intérêts et leur propre but? Dans l'activité, les tâches à accomplir sont-elles nombreuses et diversifiées? L'activité constitue-t-elle pour eux un défi à relever (ni trop facile, ni trop difficile)? L'activité débouche-t-elle sur une réalisation authentique, c'est-à-dire un'e produit/attitude/compétence qui ressemble à ce que les étudiants feront dans leur future profession? L'activité exige-t-elle un engagement cognitif (l'utilisation de stratégies d'apprentissage de haut niveau comme celles relatives à la résolution de problème)? ...). Cet effort initial devient rapidement une habitude qui aide à construire des séances plus cohérentes, plus interactives et donc plus motivantes. 


\section{Évaluation}

Dans la plupart de nos expériences d'enseignement, l'évaluation sommative était la plus couramment utilisée et souvent exclusivement. Certains outils introduits en formation, telle que la grille critériée (Berthiaume et al., 2011), nous ont permis de découvrir l'intérêt d'une évaluation formative reprenant, en cours de formation, les objectifs explicités en amont selon le concept d'alignement pédagogique et donnant plus de transparence aux étudiants sur le processus de leur évaluation.

Par la suite, nous avons varié nos méthodes d'évaluation et proposé à nos groupes d'étudiants des évaluations formatives (Daele et Berthiaume, 2011; Nicol et MacfarlaneDick, 2006) :

- sous forme d'évaluation entre pairs, ou d'auto-évaluation, avec l'aide d'une grille critériée;

- sous forme de co-construction de tests : en leur demandant de produire un grand nombre de questions parmi lesquelles certaines, au choix de l'enseignant, figurent dans le test final;

- sous forme de portfolio à réaliser tout au long de l'année.

De façon plus classique, nous avons continué à utiliser des formes d'évaluation sommative, en insistant sur l'explication de la grille critériée, présentée en amont de l'examen (qu'il soit écrit ou oral), de façon à ce que l'étudiant maitrise la façon dont il va être évalué.

Les nouvelles formes d'évaluation que nous avons mises en place dans nos enseignements témoignent de notre évolution et ont participé à notre processus de transformation. La mise en œuvre de l'évaluation s'est opérée différemment pour chacun d'entre nous, alors que l'utilisation de la grille critériée a été évidente et massive pour tous, l'évaluation entre pairs et la co-construction de tests ne se sont réalisées que partiellement, selon l'implication des étudiants.

\subsubsection{Synthèse des transformations opérées}

Nous avons tous opéré une transformation pédagogique d'un de nos cours, accompagné par une conseillère pédagogique pour les trois phases de préparation, d'observation et de debriefing. Si nous avons tous, par la suite, poursuivi une transformation de la plupart de nos cours, il nous a été demandé dans notre bilan réflexif, de nous concentrer sur la première transformation accompagnée pour en rendre compte de façon à en tirer les leçons et installer des réflexes de transformations ultérieures.

CF : Concernant ma transformation pédagogique, il s'est agi de faire le deuil du contenu (diminuer la partie "cours magistral»), de donner du sens (passer du temps à expliquer pourquoi), de prendre le temps du feedback (écouter pour mieux mesurer les apprentissages) et d'oser la co-construction du cours et des tests d'évaluation.

Ensuite, certains d'entre nous ont mis en place une évaluation globale du cours qui, soit par la forme d'un questionnaire anonyme, soit plus informellement en interrogeant sur post-it les points positifs et les suggestions d'amélioration, permet de susciter un avis constructif de la part des étudiants, source d'inspiration et d'amélioration continue.

IC : En pédagogie, j'ai découvert au travers de cette réflexion, que le souci de la forme et des techniques d'enseignement, revalorisant l'expérience de l'apprenant en interaction avec celle du 
collectif enseignant, pouvait ouvrir et inscrire chacun des acteurs dans une démarche de coconstruction de l'objet enseigné

Une fois le changement opéré et après avoir pris le recul nécessaire, en partageant cette expérience par écrit dans nos bilans respectifs et en participant aux séminaires pédagogiques proposés par SAPIENS, est venue l'envie de partager avec ses pairs, mais aussi de façon plus large, ou au sein d'une communauté de pratiques (Périsset et al., 2019) et par la publication d'un retour d'expérience. Cette même idée d'entrer dans une dynamique plus collective était exprimée unanimement en conclusion de tous nos bilans réflexifs. Au moins trois facteurs ressortent de la réflexion de chacun comme ayant favorisé l'émergence de cette énergie: le fait que nous étions au départ tous "vierges" de concepts pédagogiques et très ouverts à une disruption, l'interdisciplinarité et l'environnement relationnel constructif et inter-apprenant qu'elle permet de créer sans enjeux parasites de compétition et enfin l'effet bienfaisant du changement de posture, pour l'enseignant comme pour l'étudiant.

\subsection{Analyse réflexive issue du focus group}

L'intention était d'initier une collaboration réflexive entre enseignants-chercheurs et conseillères pédagogiques. Dans cette démarche, les conseillères pédagogiques ont emprunté la méthodologie du focus group pour proposer un temps d'échange afin que les enseignants certifiés puissent verbaliser et conscientiser leur expérience d'apprentissage dans le cadre de CertifiENS. Après une transcription détaillée du focus group, l'analyse du discours et des interactions a permis de dégager quatre thèmes :

- les intentions et les motivations à l'origine de l'engagement dans cette démarche de formation (élément déclencheur, parcours de formation antérieur, justifications apportées pour soi, les pairs et l'institution...);

- les pratiques pédagogiques effectives (avant, pendant et après le certificat, afin de saisir à partir des pratiques déclarées les transformations dans la pratique enseignante);

- l'expérience du parcours de formation CertifieNS (organisation des différents temps de travail, évolution des représentations de ses connaissances en pédagogie...);

- l'expérience de l'interdisciplinarité durant le parcours de formation (évolution des représentations quant à une formation impliquant des enseignants de différentes disciplines, perception des différences épistémologiques...).

\subsubsection{Motivations et intentions de départ}

Le constat principal est celui d'un paradoxe : malgré une représentation floue de ce que pouvait être la pédagogie universitaire ("une telle inconnue», "un peu le vide intersidéral »), tant d'un point de vue théorique que pratique, les participantes soulignent qu'elles avaient le sentiment qu'il y avait matière à un changement souhaitable dans leurs pratiques enseignantes. L'engagement dans le parcours de formation est alors décrit, non comme décision influencée par des arguments factuels (par exemple, demande institutionnelle ou constats d'échec en cours), mais plutôt comme initié par un ressenti : " on sentait qu'il y avait une transformation à l'œuvre, mais pas encore caractérisée ». Les enseignantes reconnaissent qu'il est plus intéressant d'aborder les formations à la pédagogie par le biais de la motivation 
personnelle plutôt que par obligation institutionnelle, car la réponse des institutions et universités est souvent peu en adéquation avec les besoins réels des enseignants. En effet, les institutions perçoivent l'innovation pédagogique uniquement à travers le spectre du numérique, qui reste une "coquille vide" si on ne commence pas par les bases. Le besoin de changer de paradigme est très présent chez les quatre participantes; il n'était peut-être pas identifié dès le début, malgré une forte motivation intrinsèque et une envie forte de faire évoluer sa façon d'enseigner ou du moins de se renseigner sur la question de l'enseignement.

\subsubsection{Pratiques pédagogiques effectives}

Une étape essentielle dans le changement des perceptions et de ses pratiques d'enseignement est d'être conscient de sa propre approche de l'enseignement et de l'expérience d'apprentissage des étudiants (Trigwell et Prosser, 2004). Ce travail de verbalisation de ses apprentissages pédagogiques et de l'évolution de ses pratiques d'enseignement était l'intention centrale de ce focus group et des conseillères pédagogiques. Le changement de posture des enseignantes certifiées est évident et indéniable et reflète la transformation la plus visible des pratiques pédagogiques, ainsi qu'illustré par beaucoup de verbatim dans le discours sur les opinions et représentations des participantes concernant les étudiants, les pairs enseignants et leur rôle. Cette démarche de formalisation objective de ses pratiques pédagogiques a ainsi été déclenchée et se poursuit au travers de partage d'expérience formel et informel (rédaction d'article, participation à des communautés de pratiques, des journées d'études, échanges entre collègues etc.) et a réellement permis aux enseignantes certifiées de réaliser l'ampleur des transformations pédagogiques entreprises. L'analyse du discours a clairement fait émerger que cette évolution positive des conceptions de l'enseignement et de l'apprentissage va non seulement de pair avec l'évolution des pratiques d'enseignement, mais également qu'une fois le changement de paradigme opéré, celui-ci semble irréversible et semble alors s'instaurer un cercle vertueux.

LA : Être à l'écoute de son collègue dans un atelier SAPIENS, finalement de changer de position, de laisser ses prérequis et ses préjugés de côté, se mettre dans cette posture d'être à l'écoute, ça favorise, après, en dehors de l'espace SAPIENS, ce mouvement-là avec nos étudiants.

Je ne suis plus dans la position d'enseignant et l'autre d'étudiant ou d'apprenant: il sait déjà quelque chose, je fais émerger ses connaissances antérieures et j'adapte mon cours: c'est un changement de position.

Chacune réalise aussi le chemin à parcourir, pour transformer ses enseignements et, de manière plus générale, les pratiques pédagogiques à l'université. Le changement de posture est donc vécu à la fois comme une expérience commune au sein du groupe de formation SAPIENS, mais individuelle et solitaire au sein des universités respectives de la COMUE. Les participantes reconnaissent unanimement l'enjeu énorme que représente la formation des enseignants et valorisent le format des ateliers proposés par SAPIENS qui prône la mise en situation et une approche par l'expérience; les ateliers de formation sont conçus comme une séance de cours avec des objectifs, des activités, une évaluation, ce qui permet à l'enseignant participant de vivre lui-même l'expérience d'apprentissage qu'il pourra ensuite transférer dans ses enseignements et reproduire auprès de ses étudiants. 


\subsubsection{L'expérience de l'interdisciplinarité}

Les séances de formation tout au long du parcours intègrent des enseignants de disciplines très variées. Les participantes soulignent trois aspects positifs de l'interdisciplinarité au sein des séances de formations :

- une prise de conscience de problématiques similaires et d'objectifs communs d'une discipline à une autre;

- un effet miroir intéressant permettant d'aborder la réflexion sur sa pratique sous un autre angle, à travers l'image que l'autre nous renvoie;

- la prise de conscience de la transférabilité des stratégies d'enseignement et d'apprentissage, des méthodes et des outils d'une discipline à une autre, d'une science à une autre.

En revanche, elles notent également les limites de l'interdisciplinarité et mentionnent l'aspect chronophage, le manque d'efficience ou d'efficacité immédiate et l'effort cognitif trop important que l'exercice demande parfois (de transposition d'une discipline à une autre). Finalement, une des solutions évoquées est la mise en place des communautés de pratiques par discipline.

\subsubsection{Continuer à porter le changement de paradigme}

Le terme " changement de paradigme » revient à plusieurs reprises pendant l'entretien de groupe. Il est évoqué à travers la question de la perception de l'enseignement, des méthodes et outils nécessaires à l'apprentissage de l'étudiant. Il ressort de la discussion que des formations préalables sont nécessaires pour impulser ce changement et doter les enseignants qui le souhaitent de concepts, de méthodes et d'outils pour transformer leurs pratiques et mettre des mots précis sur des besoins. Cette période de formation est l'occasion pour les enseignants d'identifier leurs pratiques actuelles et ainsi de mieux verbaliser ce besoin de changement, selon "l'état" de leur expérience personnelle et professionnelle. Pourtant, toutes les participantes s'accordent sur l'inadéquation entre les besoins des enseignants et la réponse institutionnelle apportée par leurs universités. Elles perçoivent clairement une inadéquation entre enseignants et institutions qui ne situent pas toujours l'innovation pédagogique sur le même plan épistémologique. Elles soulignent une dichotomie existante entre innovation, au sens de transformation pédagogique et de changement conceptuel (tel qu'elles la perçoivent) et, d'un autre côté, l'innovation au sens de progrès numérique, due au fait que certaines institutions privilégient leur dotation en cohorte d'outils technologiques variés pouvant rester à l'état de "gadget " si les intentions pédagogiques ne sont pas clairement définies en amont. La discussion dénonce ainsi une approche technocentrée abusive (centration sur l'outil) sans aborder une réelle réflexion sur ses pratiques enseignantes. Les participantes expriment leur difficulté de "continuer à porter le changement de paradigme » dans un environnement institutionnel qui n'a pas encore pris la mesure de la profondeur du changement nécessaire.

\section{Discussion et perspectives}

À l'issue de l'analyse réflexive de cette expérience partagée de formation, rendue possible grâce à la conjugaison des efforts de la jeune équipe SAPIENS et de la première 
promotion d'enseignants ayant répondu à son offre de formation CertifiENS, nous proposons d'en discuter les perspectives, tant du point de vue institutionnel que dans nos contextes disciplinaires respectifs.

\subsection{Dans le cadre institutionnel}

Il semble important de décrire les éléments du paysage universitaire dans lequel SAPIENS s'est efforcé de proposer des axes de développement de sa mission d'innovation et de transformation des pratiques pédagogiques, dans le prolongement de la création de CertifiENS. En effet, en 2019, une politique de concentration de moyens universitaires en région parisienne a acté la création de l'Université de Paris, à partir de la fusion de plusieurs établissements, mais non la totalité des membres de la COMUE USPC. Cette restructuration du mode de gouvernance n'a bien sûr pas été sans impacter la dynamique d'engagement des ER en réponse à une offre de formation interdisciplinaire proposée au niveau d'une COMUE non stabilisée. Cette offre par l'équipe SAPIENS s'est néanmoins maintenue à l'adresse de l'ensemble de la COMUE USPC, devenue ASPC (Alliance Sorbonne Paris Cité) (SAPIENS - Alliance Sorbonne Paris Cité, s.d.), avec une promotion de 15 certifiés en 2019 et une promotion en cours de 12 candidats à la certification en 2020.

Afin d'approfondir la formation, l'équipe SAPIENS a proposé aux certifiés deux opportunités collectives de perfectionnement : trois séances de codéveloppement et un module hybride, dit de " perfectionnement ». Les séances de codéveloppement (Payette et Champagne, 1997) ont pour objectif, à partir d'une situation-problème amenée par un participant, de mobiliser l'intelligence collective pour faire émerger des pistes d'action claires, relancer la motivation et améliorer sa pratique pédagogique. Parallèlement, SAPIENS a créé en 2019 le dispositif de Perfectionnement (SAPIENS Formations, 2020), avec pour objectif de continuer à guider les enseignants dans leur démarche de développement professionnel, en leur proposant, en présentiel ou à distance, des temps de réflexion autour de leurs pratiques enseignantes et un espace en ligne d'apprentissage informel. Ce dispositif est avant tout une opportunité d'échanger entre pairs, de donner et recevoir des conseils et avoir accès à des ressources (documentation relative aux séances de codéveloppement, espace de partage de ressources enrichi de fiches conseils, bibliographie et annuaire collaboratifs, forum type "la machine à café »), permettant à chacun de s'investir à son rythme et en fonction de ses disponibilités. Ce dispositif est récent et mérite qu'on lui laisse le temps de faire ses preuves.

\subsection{Dans le cadre de l'insertion de chaque certifié dans son contexte}

Au sein de notre groupe de certifiés, les éléments communs de transformation sont nombreux. Nous les abordons ici, sans volonté de les hiérarchiser, ni de les catégoriser, mais plutôt de faire émerger un processus de transformation commun et donc potentiellement reproductible.

La transformation pédagogique initiée pendant le programme de formation se généralise à l'ensemble des enseignements et nous avons acquis de la légitimité pour partager nos nouvelles pratiques, accentuer des pratiques réflexives avec nos pairs et 
en favoriser la diffusion au sein de nos disciplines ou environnements respectifs. Certaines d'entre nous sont à l'origine de nouveaux enseignements, ou projets transversaux, favorisant la collaboration entre enseignants et entre étudiants. Il apparaît clairement que la diffusion et le partage entre pairs deviennent des pratiques courantes.

Les retours réguliers au sein de notre communauté de pratiques CertfiENS-SAPIENS, sous la forme d'échanges, de retours d'expériences informels, ou de séances de codéveloppement plus structurées, se font régulièrement et permettent de maintenir le niveau d'énergie ainsi qu'une motivation à poursuivre les efforts. Si nos démarches individuelles apparaissent isolées à l'échelle de nos cours, voire perturbatrices au sein de nos équipes enseignantes, nos actions de sensibilisation ont pu prendre la forme d'une assistance de nos collègues à transformer leurs cours ou d'une invitation à participer aux formations ou aux événements SAPIENS. Cependant, nous avons eu besoin d'être soutenus par nos pairs : ceci illustre le mouvement de l'individuel vers le collectif, puis du retour à l'individuel avec le collectif comme appui. Malgré l'isolement dans nos établissements respectifs, la communauté CertifiENS nous a accompagnés, par la légitimité issue de nos formations, ainsi que par le lien créé.

Le changement de posture de l'enseignant s'est manifesté pour chacun d'entre nous: pour certains, presque « manager » ou chef de chantier d'une construction de cours et sachant aussi se laisser guider par les besoins de l'étudiant ou les échanges avec les autres enseignants; pour d'autres, la période de formation a généré une mise à distance transitoire qui, dans ce processus de transformation pédagogique, nous a permis d'opérer une disruption de nos pratiques. Pour reprendre les résultats de Deschryver et Lameul (2016), nous avons opéré une remise en question de nos convictions par rapport au mode d'apprentissage des étudiants et partageons un "besoin éprouvé de questionnement partagé avec des collègues au sein d'un groupe de pairs engagés dans les pratiques innovantes ».

Le constat de l'irréversibilité de cette transformation est partagé par tous : une fois le processus de transformation enclenché, la dynamique est lancée et se poursuit. Un retour en arrière est impossible. Le vécu de cette transformation n'est pas linéaire et laisse transparaître, au fil du temps, certaines difficultés, notamment le sentiment de décalage entre notre position et celle de notre institution. L'énergie déployée par chacun de nous dans ce processus est donc parfois coûteuse, au regard des évolutions du processus que nous souhaiterions plus global, dans nos établissements.

Cette nouvelle posture conduit à plus d'investissement institutionnel et à un intérêt pour la démarche de recherche-action. Nous sommes désormais identifiés par nos institutions comme référents sur les sujets pédagogiques et moteurs pour participer à des groupes de réflexion. Dans le domaine de la recherche, nous nous inscrivons dans un projet de prototypage de pairs-accompagnateurs qui nous mène à construire un protocole de recherche. Cette synergie de l'expérience CertifiENS avec l'engagement dans un groupe de réflexion intitulé "Éthique des Curriculum en Santé » a favorisé chez l'une d'entre nous, une forme de prise de conscience de la nécessité d'expliciter les paradigmes dans lesquels viennent s'ancrer nos agir professionnels en tant que chercheurs ou enseignants, la notion de paradigme pouvant se définir en philosophie des sciences autour des trois dimensions ontologique, épistémologique et méthodologique (Parent et al., 2018). Grâce à cette prise de conscience, la transformation pédagogique opérée a pu s'ancrer plus en profondeur permettant 
d'évoluer d'une posture enseignante de transmission de connaissances, vers une posture d'accompagnement de l'apprentissage de compétences, portée dans la réforme en cours des études médicales en France. Cette nouvelle posture appelle un nécessaire dialogue des paradigmes (Guba, 1990), articulant selon la nature des compétences enseignées et les visées pédagogiques, une posture objectiviste (paradigme positiviste) ou plus subjectiviste (paradigme constructiviste).

\section{Conclusion}

La description et l'analyse réflexive de notre expérience de formation et de transformation pédagogique accompagnée par le service SAPIENS, nous ont permis d'en dégager les éléments clés qui ont conduit efficacement à la transformation en profondeur de l'ensemble de nos pratiques pédagogiques. Elles ont aussi renforcé les liens déjà établis entre nous pendant la formation, faisant émerger la dynamique collective d'échanges vivifiants sur nos pratiques et fondant la perspective d'une réelle communauté de pratiques.

Avancer dans notre parcours de formation en interdisciplinarité a eu plusieurs effets. Tout d'abord, notre motivation a évolué du simple besoin d'acquérir de nouveaux outils et méthodes vers une aspiration à plus de créativité, puisant dans la communauté de pratiques informelle constituée par l'expérience, l'énergie pour transformer nos pratiques enseignantes et notre écosystème universitaire. Ensuite, l'expérience s'est enrichie avec les allers-retours de chacun entre l'environnement universitaire de sa propre discipline, où il est devenu référent et le groupe interdisciplinaire de pairs «transformés » ensemble. L'arrivée de nouvelles promotions d'enseignants CertifiENS devrait venir enrichir et faciliter la constitution d'une communauté de pratiques plus formelle.

À l'issue du parcours CertifiENS et des transformations que nous avons décrites et analysées, notre posture professionnelle a profondément évolué. Faire l'expérience, en tant qu'apprenant en formation, du changement de paradigme pédagogique, a constitué le levier principal de cette évolution. Les concepts pédagogiques ont été structurants pour initier et accompagner ces changements, en faisant appel à nos acquis, en s'appuyant sur une dynamique d'attentes partagées et sur l'esprit collaboratif. Chacun a puisé dans cette expérience de formation selon ses besoins, pour en mobiliser ensuite les concepts dans sa pratique, prenant parfois avec l'expérience, un peu de distance. Comme le soulignent Lison et Paquelin, "plus qu'une formation, l'enjeu est davantage celui du développement professionnel des enseignants dans une perspective d'amélioration continue des pratiques ancrée dans une approche systémique » (Lison et Paquelin, 2019). 


\section{BIBLIOGRAPHY}

Basque, J. (2007). L'élaboration du scénario pédagogique. Texte tiré du cours EDU 1030. Télé-université de Québec. http://www.teluq.ca/edu1030

Berthiaume, D., David, J. et David, T. (2011). Réduire la subjectivité lors de l'évaluation des apprentissages à l'aide d'une grille critériée : Repères théoriques et applications à un enseignement interdisciplinaire. Revue internationale de pédagogie de l'enseignement supérieur, 27(27(2)), Article 2. https://doi.org/10.4000/ripes.524

Biggs, J. (1996). Enhancing teaching through constructive alignment. Higher Education, 32(3), 347-364. https://doi.org/10.1007/BF00138871

Brauer, M. (2011). Le syllabus (ou « descriptif de cours »). In Enseigner à l'université : Conseils pratiques, astuces, méthodes pédagogiques. Armand Colin.

Casey, K. (1995). Chapter 5 : The New Narrative Research in Education. Review of Research in Education, 21(1), 211-253. https://doi.org/10.3102/0091732X021001211

Daele, A. et Berthiaume, D. (2011). Choisir ses stratégies d'évaluation. Université de Lausanne. https://www.unil.ch/files/live/sites/cse/files/shared/brochures/ memento_m4_strategies_evaluation_V3_13fevrier2011.pdf

Daele, A. et Berthiaume, D. (2013). Chapitre 6. Comment structurer les contenus d'un enseignement? In D. Berthiaume et N. Rege-Colet, La pédagogie de l'enseignement supérieur : Repères théoriques et applications pratiques. Tome 1 : Enseigner au supérieur. Peter Lang $\mathrm{CH}$. https://doi.org/ 10.3726/978-3-0352-0230-4

De Ketele, J.-M., Chastrette, M., Cros, D., Mettelin, P. et Thomas, J. (2007). Section 1. La technique des objectifs pédagogiques. Dans Le guide du formateur (3éd., p. 95-111). De Boeck Supérieur.

De Ketele, J.-M. et Roegiers, X. (2015). Méthodologie du recueil d'information. Fondements des méthodes d'observations, de questionnaires, d'interviews et d'études de documents. De Boeck Supérieur.

Deschryver, N. et Lameul, G. (2016). Vers une opérationnalisation de la notion de posture professionnelle en pédagogie universitaire. Revue internationale de pédagogie de l'enseignement supérieur, 32(3). https://doi.org/10.4000/ripes.1151

Duchesne, S. et Haegel, F. (2004). L'enquête et ses méthodes : Les entretiens collectifs. Nathan.

Guba, E. G. (éd.). (1990). The Paradigm dialog. Sage Publications.

Herda, E. A. (1999). Research conversations and narrative : A critical hermeneutic orientation in participatory inquiry. Praeger.

Journal officiel de la République française, Décret n 2017-854 du 9 mai 2017 modifiant le décret $\mathrm{n}^{\circ}$ 84-431 du 6 juin 1984 modifié fixant les dispositions statutaires communes applicables aux enseignants-chercheurs et portant statut particulier du corps des professeurs des universités et du corps des maîtres de conférences (2017). https://www.legifrance.gouv.fr/loda/id/ JORFTEXT000034632129/

Lalle, P. et Bonnafous, S. (2019). La révolution pédagogique de l'enseignement supérieur, une universalité géographique et paradigmatique : Introduction. Revue internationale d'éducation de Sèvres, 80, 49-60. https://doi.org/10.4000/ries.8142 
Lison, C. et Paquelin, D. (2019). La formation du supérieur : Un levier de transformation des universités québécoises. Revue internationale d'éducation de Sèvres, 80, 61-70. https://doi.org/ $10.4000 /$ ries. 8184

Mager, R. F. (1972). Comment définir des objectifs pédagogiques. Gauthier-Villars.

$M O O C$ «Se former pour enseigner dans le supérieur ». (s.d.). FUN MOOC. Consulté 24 avril 2021, à l'adresse http://www.fun-mooc.fr/fr/cours/se-former-pour-enseigner-dans-le-superieur/

Moyle, K. (2007). Focus groups in educational research : Using ICT to assist in meaningful data collection. Proceedings of the International Research Conference of the Australian Association for Research in Education 2006, 1-12. https://researchprofiles.canberra.edu.au/en/publications/focusgroups-in-educational-research-using-ict-to-assist-in-meani

Musial, M., Pradère, F. et Tricot, A. (2012). Comment concevoir un enseignement? De Boeck. Nadeau, M.-A. (1988). Les objectifs pédagogiques. Dans L'évaluation de programme: Théorie et pratique (p. 251-284). Presses de l'Université de Laval.

Nicol, D. J. et Macfarlane-Dick, D. (2006). Formative assessment and self-regulated learning : A model and seven principles of good feedback practice. Studies in Higher Education, 31(2), 199-218. https://doi.org/10.1080/03075070600572090

Parent, F., Aiguier, G., Berkesse, A., Reynaerts, M., Rolland, F., Wardavoir, H. et Jouquan, J. (2018). Penser l'éthique des curriculums de formation professionnelle en santé au regard d'une perspective épistémologique de « l'agir-en-santé ». Pédagogie Médicale, 19(3), 127-135. https:// doi.org/10.1051/pmed/2019020

Payette, A. et Champagne, C. (1997). Le groupe de codéveloppement professionnel. Presses de l'Université du Québec.

Périsset, D., Vuillet, Y. et Barras, H. (2019, janvier 8). Constitution d'une communauté de pratique de formateurs du supérieur à travers la co-construction d'un outil : La matrice d'analyse de l'activité didactique et pédagogique d'enseignant.e.s en formation à l'enseignement secondaire (HEP-VS). Colloque ADMEE, Lausanne.

Pratiques pédagogiques avec le numérique. (s.d.). éduscol | Ministère de l'Éducation nationale, de la Jeunesse et des Sports - Direction générale de l'enseignement scolaire. https:// eduscol.education.fr/157/pratiques-pedagogiques-avec-le-numerique

SAPIENS - Alliance Sorbonne Paris Cité. (s.d.). SAPIENS - Alliance Sorbonne Paris Cité. Consulté 24 avril 2021, à l'adresse https://sapiens-uspc.com/

SAPIENS - Formations. (2020, mars 30). SAPIENS - Formations. https://sapiens-uspc.com/ formations/

Taddéi, F. (2018). Apprendre au XXI ${ }^{e}$ siècle. Calmann-Lévy.

Touraine, A. (1978). La voix et le regard. Seuil.

Trigwell, K. et Prosser, M. (2004). Development and Use of the Approaches to Teaching Inventory. Educational Psychology Review, 16(4), 409-424. https://doi.org/10.1007/s10648-004-0007-9

Vermersch, P. (2017). L'entretien d'explicitation. ESF.

Viau, R. (1999). La motivation dans l'apprentissage du français. ERPI.

Wenger, E. (1998). Communities of practice : Learning, meaning, and identity. Cambridge University Press. 


\section{NOTES}

1. Nous retenons ici l'expression anglo-saxonne focus group plutôt que la version française « entretien collectif » pour insister sur le fait que l'entretien était centré sur une expérience précise (la participation au certificat de pédagogie) et concernait un groupe d'enseignants déjà constitué, justement autour de l'expérience du certificat, et non pas un rassemblement d'acteurs ne se connaissant pas. (Duchesne et Haegel, 2004)

\section{ABSTRACTS}

This article is the result of a reflective collaboration between five university teachers from different disciplines and universities, with the help of two pedagogical advisors. It consists of feedback following the participation of these teachers in a training course in university pedagogy entitled CertifiENS. Our purpose is to describe this original and interdisciplinary experience, by describing the characteristics of our group of teachers, the CertifiENS program that we followed and pedagogical transformations that we made in our teaching. We asked two of our pedagogical advisors (instructors in the CertifiENS program and teacher-researchers in sociology and education sciences) for a focus group interview to uncover our initial intentions and motivations and to highlight the emergence of a collective dynamic. This focus group interview helped us to carry out an individual and then collective reflective analysis of the individual transformation process and of the pedagogical transition that had taken place and is still in progress.

This analysis showed that interdisciplinarity was a major asset and a source of synergies, allowing us to focus on the common ground: pedagogy. The group was the bearer of a sustainable transformation of teaching practices and beliefs, each one on their respective field wished to continue to carry this paradigm shift, while advancing on the construction of a community of practice.

Cet article est le fruit d'une collaboration réflexive de cinq enseignants de champs disciplinaires et de structures universitaires différents, avec l'aide de deux conseillères pédagogiques. Il s'agit d'un retour d'expérience à la suite de la participation de ces enseignants à un parcours de formation à la pédagogie universitaire intitulé CertifiENS. Notre propos est surtout de témoigner de cette expérience originale et interdisciplinaire, en décrivant les caractéristiques de notre groupe d'enseignants, le parcours CertifiENS que nous avons suivi puis les transformations pédagogiques que nous avons opérées dans nos enseignements. Nous avons sollicité a posteriori deux de nos conseillères pédagogiques (formatrices dans le cadre du parcours CertifiENS et enseignantes-chercheuses en sociologie et sciences de l'éducation) pour un entretien de groupe (focus group) visant à expliciter nos intentions et motivations initiales et mettre en lumière l'émergence d'une dynamique collective. Cet entretien de groupe nous a permis de porter une analyse réflexive individuelle puis collective sur le processus de transformation individuelle et sur la transition pédagogique effectuée et toujours en devenir.

Cette analyse a montré que l'interdisciplinarité a été un atout majeur et une source de synergies, permettant de nous focaliser sur le commun: la pédagogie. Le groupe a été porteur d'une transformation durable des pratiques enseignantes, chacun sur son terrain respectif a souhaité continuer à porter ce changement de paradigme, tout en réfléchissant à la construction d'une communauté de pratiques. 
INDEX

Mots-clés: posture de l'enseignant, interdisciplinarité, transformation pédagogique, retour d'expérience, analyse réflexive

\section{AUTHORS}

\section{CATHERINE DE LA FOUCHARDIÈRE}

Maître de conférences en marketing, Université Sorbonne Paris Nord, France - catherine.de-lafouchardiere@univ-paris13.fr

\section{LAURE ABIAD}

Chargée de cours en langue, Université Paris 3 Sorbonne Nouvelle, France, laure.abiad@sorbonne-nouvelle.fr

\section{MOUSSA LAANANI}

Assistant hospitalo-universitaire en biostatistiques et épidémiologie, Université Paris-Descartes, Institut national de la santé et de la recherche médicale (Inserm), France, moussa.laanani@inserm.fr

\section{MARINE LANTERI}

Conseillère pédagogique, Université de Paris, France, marine.lanteri@uspc.fr

\section{MORGANE MARIDET}

Conseillère pédagogique, Université Paris 3 Sorbonne Nouvelle, France, morgane.maridet@sorbonne-nouvelle.fr

\section{ANNABELLE TENENBAUM}

Maître de Conférence Universitaire - Praticien Hospitalier, UFR d'Odontologie-Garancière, Université de Paris, France.Laboratoire Éducations et Pratiques de Santé, UR 3412, Université Sorbonne Paris Nord, F-93430, Villetaneuse, France, annabelle.tenenbaum@u-paris.fr

\section{ISABELLE COLOMBET}

MCU-PH, Médecin en Équipe Mobile de Soins Palliatifs Hôpital Cochin, Assistance Publique Hôpitaux de Paris - Enseignant Chercheur, UFR Médecine, Université de Paris, France, isabelle.colombet@u-paris.fr 